\title{
A two-stage detection model applied to skilled visual search by radiologists
}

\author{
RICHARD G. SWENSSON \\ Harvard Medical School and Peter Bent Brigham Hospital, Boston, Massachusetts 02115
}

\begin{abstract}
The model treats the detection of targets in a visual search task as a concatenation of two serial detection stages. Preattentive visual mechanisms in the initial stage function as a filter, selecting specific features of a visual pattern for the observer's explicit attention and final cognitive evaluation. The model uses bivariate normal distributions to represent the decision variables for the two serial stages, assuming different parameters for the target and nontarget features in a test set. The model is applied to the detection performance of radiologists interpreting chest $x$-rays under various conditions of search. It accounts for the substantial improvement in radiologists' ability to distinguish between target and nontarget test features when they had to search the x-ray images, compared to their performance without visual search. A change in the ROC curve between two different search tasks could be interpreted as a shift in the selection cutoff used by the preattentive filter.
\end{abstract}

Visual search tasks performed by skilled human observers often need to be treated as complex problems in signal detection. As an example, consider the detection problem confronted by a radiologist in interpreting a chest radiograph, the most common type of x-ray examination. The radiologist must search the pattern of silhouettes formed by the $x$-ray attenuations of many superimposed anatomical structures for any of a large and heterogeneous set of potentially abnormal features (targets). An individual image is a highly complex pattern that always contains a great many normal (nontarget) features, and may include several coexisting target abnormalities. Since variations in normal x-ray patterns may mimic characteristics of actual pathological conditions, the radiologist of ten cannot be certain whether or not a particular radiographic feature represents an actual target.

The detection process in such a complex task involves at least two logically sequential stages. To report a target, a radiologist must consider a specific pattern feature and decide that it cannot be interpreted as part of the normal anatomy. This final decision stage must logically follow some earlier stage of visual search, during which that particular feature of the pattern was selected for attention and specific consideration. A distinction between separate perceptual-recognition and decision-making stages in radiographic interpretation has also been suggested by analyses of eye movements and fixa-

This research was supported by USPHS Grants GM 18674 and GM 23891. I thank Samuel J. Hessel and Peter G. Herman, who collaborated in obtaining the empirical data presented here, and Martin Deacutis, who wrote the computer program to calculate the ROC curves predicted by the model. tion latencies, recorded while observers searched radiographic images (Kundel, Nodine, \& Carmody, 1978). These serial stages of processing recall Neisser's (1967) theoretical distinction between "preattentive" and "focal attention" processes in visual search. They are also closely related to the familiar concept of hierarchically organized "feature detection" and "feature classification" processes in perception. Several recent formal models have applied similar concepts to obtain predictions about latency and accuracy of search for targets in displays of symbolic visual material (Harris, Shaw, \& Bates, 1979; Hoffman, 1978).

The model for visual search proposed here assumes that a skilled observer has developed an efficient set of automatic visual mechanisms for searching familiar types of visual patterns. These visual mechanisms are assumed to function "in parallel" as an initial filter, which selects a subset of the features in a particular pattern for the observer's attention and specific consideration. Each selected feature receives an explicit evaluation by a cognitive process which determines whether (and at what level of confidence) that feature will be reported as a target.

The next section uses concepts of signal detection theory to formalize these ideas as a model for a twostage detection system. The model postulates a concatenation of two serial decision processes, in which the output of the first process (the preattentive filter) provides the input to the second (the cognitive evaluation). The model's parameters, which specify the detection performance of this two-stage system, characterize the detection capabilities of the separate search and evaluation stages and the dependence between these stages. In general, the two stages of 
detection will not be independent, because the physical characteristics used to select pattern features for visual attention in a particular situation are likely to overlap those used to evaluate how closely a selected feature resembles a target upon specific consideration. The model's free parameters can be reduced by specific assumptions and estimated from independent sets of data collected in several different experimental conditions. The model's application is illustrated by its fit to data from two separate series of experiments that studied the interpretation of chest radiographs by skilled radiologists.

\section{THE GENERAL SEARCH MODEL}

To be formally explicit, the model assumes the existence of some "test set" composed of specific target and nontarget features within a sample of visual patterns of a type familiar to the observer. The model further assumes that each individual feature in the test set can be characterized by the value of two random variables, $\mathrm{X}$ and $\mathrm{Y}$, for a given observer. The variable $X$ represents the feature's "salience," as measured by the observer's preattentive filter, and Y represents the decision variable measured by his cognitive evaluation stage. In a search task, the observer is assumed to establish a salience criterion (C) for his preattentive filter, such that a given feature is selected for attention only if $\mathrm{X}>\mathrm{C}$. The final decision output for each selected feature depends upon the value of $Y$. The model assumes the observer uses an ordered set of decision cutoffs, $R_{l}, \ldots, R_{m}$, to classify each selected feature into one of $m+1$ reporting categories, such that the feature is assigned to Category 0 (not reported) if $Y \leqslant R_{1}$, to Category $j(j=1, \ldots, m-1)$ if $R_{j}<Y \leqslant R_{j+1}$, and to Category $m$ (highest confidence target) if $Y>R_{m}$. Thus, a given test feature will be reported at or above the $j^{\text {th }}$ confidence category only if both $\mathrm{X}>\mathrm{C}$ and $\mathbf{Y}>\mathbf{R}_{\mathbf{j}}$ for that feature.

The performance of any detection system can be described by its receiver operating characteristic (ROC) curve (Green \& Swets, 1974). The ROC curve indicates how $1-\beta$ and $\alpha$, the probabilities of true and false detections (conditional on target and nontarget events), covary from near zero to near unity as the criterion for making a detection decision changes from very stringent to very lax. The theoretical form of the ROC curve depends upon the distributions assumed to represent the underlying decision variable produced by the target and nontarget events. A common assumption, often theoretically or empirically justifiable, is that these decision variables are normally distributed. Then the ROC curve becomes a linear function in normal-deviate coordinates-i.e., when the true and false detection rates are each represented by the deviate of the standard normal distribution that yields a lower-tail area equal to the given probability. Under these distributional assumptions, the slope and location parameters of empirically generated ROC curves can be estimated by using a maximum-likelihood fitting procedure (Dorfman \& Alf, 1969; Grey \& Morgan, 1972; Sandor \& Swensson, 1978).

The performance predicted by the present model is defined as the joint probability that $\mathrm{X}>\mathrm{C}$ and $\mathbf{Y}>\mathbf{R}_{\mathbf{j}}$, given the sets of target and nontarget test features. To represent the dependence between these two decision variables as simply as possible, the model assumes that $\mathrm{X}$ and $\mathrm{Y}$ are jointly distributed as a bivariate normal random variable with different parameters for the subsets of target and nontarget test features. There is no generality lost in assuming that the arbitrary scales of $\mathrm{X}$ and $\mathrm{Y}$ are each adjusted to produce a zero mean and unit standard deviation for the nontarget test features. ${ }^{1}$ Then $\mathrm{X}$ and $\mathrm{Y}$ have a standard bivariate normal distribution among nontargets, with correlation parameter $r_{n}$. The five parameters of the bivariate normal distribution for target test features are the means $\left(\Delta_{x}\right.$ and $\left.\Delta_{y}\right)$ and standard deviations ( $\sigma_{\mathrm{x}}$ and $\sigma_{\mathrm{y}}$ ) of the two marginal normal distributions of $\mathrm{X}$ and $\mathrm{Y}$, and the correlation parameter $r_{t}$.

The pairs of parameters, $\left(\Delta_{x}, \sigma_{x}\right)$ and $\left(\Delta_{y}, \sigma_{y}\right)$, specify the linear (marginal) ROC curves that would be produced in normal-deviate coordinates if only the preattentive filter or only the cognitive evaluation were used to discriminate between the target and nontarget test features, under various settings for their respective decision cutoffs. The means, $\Delta_{x}$ and $\Delta_{y}$, are the location parameters of the linear ROC curves for the two separate processes; each represents the absolute value of the normal deviate for the false-positive rate at the point when $50 \%$ of the target features would be detected by that process. The linear slopes of these marginal ROC curves would be given by $\left(1 / \sigma_{x}\right)$ and $\left(1 / \sigma_{y}\right)$ for the filter and evaluation processes, respectively.

The true and false detection rates predicted by the model can be obtained for any values of $C$ and $R_{j}$ as follows. Let $U$ and $V$ have a standard bivariate normal distribution (each marginally normal with zero mean and unit standard deviation) and correlation parameter $r$. Then $L(h, k, r)$, the joint probability that $U>h$ and $V>k$, is given by:

$$
\mathrm{L}(\mathrm{h}, \mathrm{k}, \mathrm{r})=\mathrm{Q}(\mathrm{h}) \mathrm{Q}(\mathrm{k})+\sum_{\mathrm{n}=0}^{\infty}\left|\frac{\mathrm{f}^{(\mathrm{n})}(\mathrm{h}) \mathrm{f}^{(\mathrm{n})}(\mathrm{k})}{(\mathrm{n}+1) !}\right| \mathrm{r}^{\mathrm{n}+1},
$$

where $Q(h)$ and $Q(k)$ are the upper-tail areas of the standard univariate normal distribution above the respective deviates $h$ and $k$, and where $f(n)(h)$ and $f^{(n)}(k)$ are the $n^{\text {th }}$ derivatives of the standard normal 
density function evaluated at $\mathrm{h}$ and $\mathrm{k}$, respectively (Zelen \& Severo, 1968, Equation 26.3.29). Since X and $\mathrm{Y}$ are defined to have a standard bivariate normal distribution for nontarget features, the false positive rate $(\alpha)$ is obtained by setting $h=C$, $k=R_{j}$, and $r=r_{n}$. The true positive rate $(1-\beta)$ is obtained by setting $r=r_{t}$ and rescaling the two decision cutoffs as standardized deviates of the bivariate distribution of $\mathrm{X}$ and $\mathrm{Y}$ for the set of target features: $h=\left(C-\Delta_{x}\right) / o_{x}$ and $k=\left(R_{j}-\Delta_{y}\right) / o_{y}$.

In general, this model requires seven parameters to specify the ROC curve: three for the preattentive filter $\left(\Delta_{\mathrm{x}}, \sigma_{\mathrm{x}}\right.$, and $\left.\mathrm{C}\right)$, two for the cognitive evaluation $\left(\Delta_{y}\right.$ and $\left.\sigma_{y}\right)$, and the two correlations $\left(r_{t}\right.$ and $\left.r_{n}\right)$ between these processes for the target and nontarget test features. The predicted ROC curve is generated from the pairs of true and false positive detection rates obtained for various values of $\mathbf{R}_{\mathbf{j}}$. These values can be calculated from Equation 1 by a computer program that uses polynomial approximations for $Q(h)$ and $Q(k)$ and a finite series of terms to approximate the infinite sum. ${ }^{2}$

\section{EMPIRICAL CONSIDERATIONS}

To make the model empirically useful, the number of its free parameters must be reduced, either by direct assumption or by using independent procedures for estimating some parameters. One obvious constraint to impose is that $r_{t}=r_{n} \equiv r$, which assumes that the two decision stages are related in the same way for both target and nontarget test features. Other constraints correspond to specific assumed values for certain of the model's parameters. Because the bivariate normal representation uses only a single parameter to characterize the dependence between $\mathrm{X}$ and $Y$ for each type of test feature, the assumption that $r_{t}=0$ or $r_{n}=0$ (or both) is equivalent to assuming that the two detection stages are independent for those test features, and considerably simplifies Eq. 1. The assumption that $\sigma_{\mathrm{x}}=1.0$ or $\sigma_{y}=1.0$ implies that the particular decision variable (X or $\mathrm{Y}$ ) has the same standard deviation for both target and nontarget test features, leading to a singleparameter representation for that marginal ROC curve: $\mathrm{d}_{\mathrm{x}}^{\prime} \equiv \Delta_{\mathrm{x}}$ or $\mathrm{d}_{\mathrm{y}}^{\prime} \equiv \Delta_{\mathrm{y}}$.

Another approach to constraining the model's parameters is to measure performance for the same test set in several different experimental conditions, designed to bypass the observer's perceptual filter or to vary its selection cutoff. Independent estimates of the parameters $\Delta_{\mathrm{y}}$ and $\sigma_{\mathrm{y}}$ could be obtained by fitting the ROC curve produced by an application of the final cognitive-evaluation stage alone. This requires making the assumption that the information generated within the process of evaluation $(Y)$ does not depend upon how the feature gets selected for atten- tion, whether by the observer's own preattentive processes $(X>C)$ or by some independent method of specification. Under this assumption, the ROC curve for the final evaluation stage can be estimated from within a separate "nonsearch" condition that bypasses the filter and specifies each individual target and nontarget test feature for an explicit evaluation by the observer.

If it can be assumed that the observer reports all features selected by his perceptual filter (i.e., that $\left.R_{1} \rightarrow-\infty\right)$, then the only unreported target and nontarget test features are those for which $X \leqslant C$. This means that the highest point on the model's predicted ROC curve, corresponding to the distinction between reported and not-reported test features, also represents a point on the marginal ROC curve for the perceptual filter. For this point, Equation 1 simplifies to the single term $\mathrm{L}(\mathrm{h},-\infty, \mathrm{r})=\mathrm{Q}(\mathrm{h})$, where $\alpha=$ $\mathrm{Q}(\mathrm{c})$ and $1-\beta=\mathrm{Q}\left[\left(\mathrm{C}-\Delta_{\mathrm{x}}\right) / \sigma_{\mathrm{x}}\right]$. Since each search condition estimates only a single point on the marginal ROC curve for the filter stage, data from a single condition would be sufficient to specify this curve only if $o_{\mathrm{x}}$ were known or assumed to have some fixed value (e.g., $\sigma_{\mathrm{x}}=1.0$ ). However, both $\Delta_{\mathrm{x}}$ and $\sigma_{\mathrm{x}}$ could be estimated using the data obtained from two separate search conditions, provided that: (1) all test features selected by the filter were reported in both conditions, and (2) the value of $\mathrm{C}$ changed sufficiently between conditions. Under these assumptions, the four parameters, $\Delta_{\mathrm{x}}, \sigma_{\mathrm{x}}, \mathrm{C}_{1}$, and $\mathrm{C}_{2}$, would be specified by the four independent equations for the predicted detection probabilities.

\section{APPLICATION OF THE MODEL}

This section illustrates the application of the twostage search model to data from two series of experiments that studied the detection performance of radiologists interpreting chest radiographs under various conditions of search. The two series of experiments used different groups of radiologists and measured their detection performance with respect to different types of test sets of target and nontarget features on selected $\mathrm{x}$-ray films.

\section{Experimental Methods}

General radiographic detection. One series of experiments (Swensson, Hessel, \& Herman, 1977, Note 1) studied the detection of a heterogeneous test set of chest abnormalities. The abnormal test features comprised a wide range of chest pathology on 27 selected radiographs: 47 subtle, but significant, conditions that would affect the individual patient's health or treatment. The $\mathbf{8 2}$ nontarget features in the test set were artifacts, normal variants, and superimposed normal structures, selected because they resembled significant abnormal conditions of various types. Eight radiologists interpreted the films in each of three separate conditions. In the free-search task, the observer reported all the abnormal findings he detected on each radiograph. In the focused-search task, he reported only the abnormal findings 
within certain designated areas of each radiograph, which included all 129 test features. In the nonsearch task, he evaluated each feature in the test set (targets and nontargets), specified by the nature of the alleged abnormality and its precise location on the radiograph. Every test feature was assigned to one of four categories in the search tasks (reported with high, medium, or low confidence, or "not reported") and to one of six categories in the nonsearch task ("target" or "nontarget" with high, medium, or low confidence).

Detection of pulmonary nodules. Another series of experiments (Swensson, Hessel, \& Herman, 1979) studied the detection of pulmonary nodules, a single homogeneous type of target abnormality. The target features in the test set were 48 simulated pulmonary nodules, produced by superimposing the density distributions of spherical objects at selected locations in the lung fields of 48 different normal and abnormal chest radiographs. The nontarget test features were defined by 222 specific locations in the lung fields of these same 48 radiographs, which contained superimposed normal structures that resembled pulmonary nodules.

Six radiologists interpreted each radiograph twice, once with and once without the simulated nodule, in each of two separate search tasks. The initial free-search task disguised any particular concern with pulmonary nodules by mixing the 96 test-film presentations with an additional 48 radiographs containing other types of pathology and by having the observers report all abnormal findings on each film. In addition to giving a confidence rating for each reported finding, the observer specified the position of each localized finding (including nodules) on a superimposable grid. In the nodule-search task, the observers reported only pulmonary nodules, omitting all other pathology on the radiographs; they sought to avoid missing any nodules by reporting even low-confidence possibilities. In a final nonsearch condition, each target and nontarget feature in the test set was specified by its location on the radiograph; the observer rated his confidence that each test location contained a target (nodule).

\section{Fits of the Search Model}

The confidence ratings that the observers assigned to the target and nontarget features of the relevant test set yielded an ROC curve for each experimental condition. This paper presents only the group ROC curves, generated from the pooled ratings of all observers, which were reasonably representative of ROC curves for the individual radiologists in these experiments. Figures 1 and 2 show the observed data points on the group ROC curves for each of the two series of experiments, plotted in normal-deviate coordinates. Notice that the discrimination between target and nontarget test features was considerably better in the search tasks (circular points) than in the corresponding nonsearch condition (triangular points). This difference was consistent for all individual radiologists in both series of experiments. With the heterogeneous test set (Figure 1), the group detection performance was similar in the free-search and focused-search conditions, and individual radiologists displayed no consistent differences between the two conditions. In the experiments with the homogeneous nodule test set, both group and individual performances were better when the radiologists searched for any abnormal conditions (free search) than when they searched only for pulmonary nodules (nodule search).

In fitting the search model, the nonsearch conditions were assumed to bypass the radiologists' initial, preattentive filter without affecting the explicit cognitive evaluation of each test feature. Thus, the ROC curve in the nonsearch condition measured the marginal ability of this final stage to discriminate between the target and nontarget test features. Estimates of

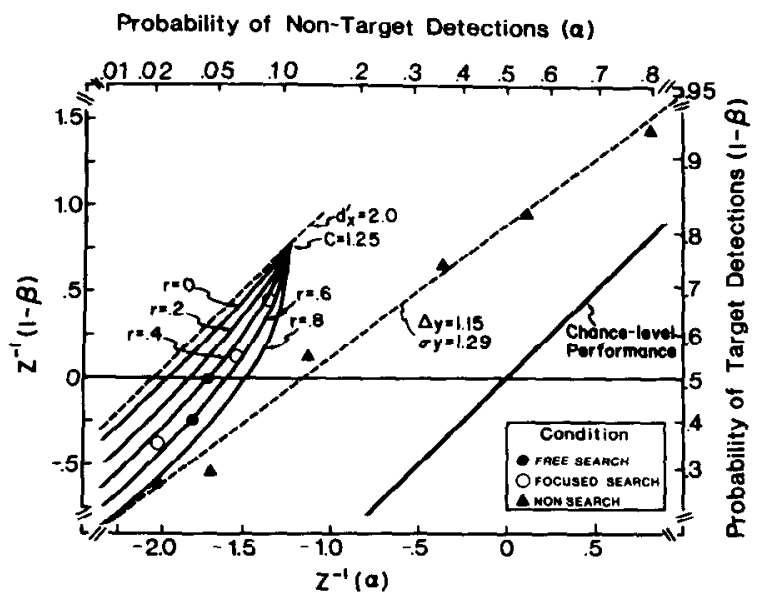

Figure 1. ROC curves for group ratings of the heterogeneous test set by eight radiologists in three separate conditions, plotted in normal-deviate coordinates. The lower broken line shows the maximum-likelihood linear fit to data from the nonsearch condition, assumed to estimate the marginal ROC curve of the model's final evaluation stage. The upper broken line with unit slope represents the marginal ROC curve assumed for the model's initial filter stage $\left(d_{x}^{\prime}=\mathbf{2 . 0}\right)$. The solid curves show the model's predicted search ROC curves for $C=1.25$ and $r=0, .2, .4, .6$, and .8 .

Probability of Non-Target Detections (a)

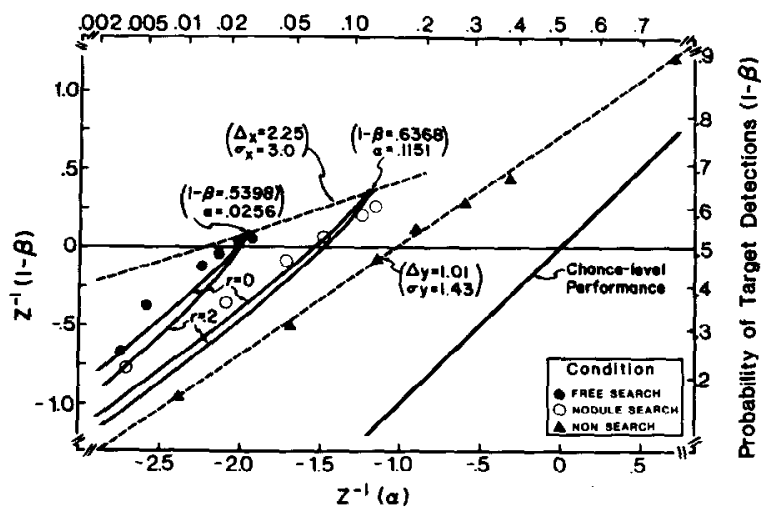

Figure 2. ROC curves for ratings of the pulmonary-nodule test set by six radiologists in three different conditions, plotted in normal-deviate coordinates. The lower broken line shows the maximum-likelihood linear fit to data from the nonsearch condition, assumed to estimate the marginal performance of the model's final evaluation stage. The upper broken line is defined by two separate points (given by the specified values of $1-\beta$ and $\alpha$ ), close to the group free-search and nodule-search performance obtained for all reported test locations. These two points were assumed to estimate the probabilities that target and nontarget test features would be selected by the model's initial filter in each condition, which then defined the filter's marginal ROC curve $\left(\Delta_{x}\right.$ $\left.=2.25, \sigma_{x}=3.0\right)$ Solid curves assume that $r_{t}=r_{n} \equiv r$ and show the model's predictions within each search condition $(C=1.95$ and $C=1.20$ ) for $r=0$ and $r=.2$. 
the linear parameters $\Delta_{\mathrm{y}}$ and $\sigma_{\mathrm{y}}$, assumed to specify this marginal performance for each test set, were obtained using a maximum-likelihood procedure; these marginal ROC curves are shown by the broken straight lines fit to the triangular data points in Figures 1 and 2.

The solid curves in Figure 1 show the ROC curves predicted by the model for various values of the correlation parameter $r_{t}=r_{n} \equiv r$, when the preattentive filter is assumed to be characterized by the single parameter $\mathrm{d}_{\mathrm{x}}^{\prime}=2.0$ (i.e., $\sigma_{\mathrm{x}}=1.0$ ) and operating at the selection cutoff $C=1.25 .^{3}$ The shape of the predicted curve depends upon $r$; it becomes more concave as $r$ increases, approaching the assumed marginal performance of the cognitive evaluation stage faster as the final evaluation rejects more and more of the originally selected features. The predicted curves for all values of $r$ end at the same point on the marginal ROC curve assumed for the filter, which corresponds to the probabilities that $\mathrm{X}>\mathrm{C}$ for both target and nontarget test features. A change in $C$ slides this end-point along the filter's assumed ROC curve, shifting the search ROC curve predicted for a given $d_{x}^{\prime}$ and $r$ without much effect on its shape. Although various assumed combinations of $d_{x}^{\prime}$ and $C$ would predict virtually indistinguishable ROC curves, the search data in Figure 1 require the model to assume that $\mathrm{d}_{\mathrm{x}}^{\prime}>1.8, \mathrm{C}<1.4$, and $\mathrm{r}>.4$ for this particular test set.

The model's predicted ROC curves in Figure 2 assume that: (1) virtually all test features selected by the radiologists" "nodule filter" were reported at some level of confidence, and (2) the filter's selection criterion (C) became more lax in the nodule-search task. The upper broken line shows the filter's marginal ROC curve, as inferred from two assumed points (specified by the values of $1-\beta$ and $\alpha$ given in Figure 2) that are close to the total proportions of reported target and nontarget test locations in each search task. Given the observed level of performance in the nonsearch task and the constraint that $r_{t}=r_{n}$, the search ROC curves have slopes that are too shallow to be closely fit by the model, particularly within the nodule-search condition. Figure 2 shows that (assuming $r_{t}=r_{n} \equiv r$ ) the model's fit is best under the assumption of independence $(r=0) .{ }^{4}$ This would mean that the physical characteristics important in searching radiographs for the locations of potential nodules were quite different from those the radiologists used in evaluating how much an individual test feature resembled a pulmonary nodule upon specific consideration.

\section{DISCUSSION}

The model presented here formalizes the idea that skilled observers can develop and apply visual strategies which avoid the need for an exhaustive evaluation of every pattern feature that might represent a target. The major function of an initial screening procedure (or "preattentive filter") would be to reduce the observer's search time by limiting the number of features that required an individual, timeconsuming evaluation. This property of a two-stage search procedure would often prove useful, even if the initial filter provided relatively poor differentiation between target and nontarget features. Whenever the final evaluation discriminates better than the filter, its marginal ROC curve lies above the filter's, and the system's detection performance improves as more and more features receive an explicit evaluation. Such a system might make relatively few false-positive detections; a stringent setting for the filter's selection cutoff would speed the process of search, but would reduce its accuracy by increasing the rate of omission errors. This pattern of results is typically found in speeded search tasks that use symbolic visual material-that is, the types of tasks for which sequential stages of visual search have been proposed (Harris, Shaw, \& Bates, 1979; Hoffman, 1978; Neisser, 1967).

However, it is also possible to envision a two-stage system whose initial filter has better detection capability than the evaluation stage that follows it. Such a two-stage system would not only be faster than a specific evaluation of all potential target features, but would also achieve superior differentiation between the target and nontarget features in any test set. In this way, the present model can account for the rather counterintuitive result that radiologists detected abnormal features much better when they had to search the radiographs than when no search was required. The model must assume that the initial (filter) stage of search provided better differentiation between the target and nontarget features of each test set than did the final evaluation stage, as measured by the explicit "yes-no" judgments about each test feature. Thus, according to the model, a major component of the radiologist's skill must reside in the "preattentive" visual mechanisms which automatically select particular features of a radiograph for his explicit attention but which apparently cannot be applied once attention is directed to a specific feature of the radiograph.

Visual search is often an integral part of the radiologist's interpretation procedure, but the precise search requirement varies considerably with the specific clinical situation-i.e., the purpose of the examination and the radiologist's prior knowledge about the patient. In many cases, the radiologist needs to search the entire radiograph for any abnormalities present (free search); however, the clinical situation may direct him to consider a specific anatomical area (focuscd search), a more-narrow set of target abnormalities (e.g., nodules), or simply certain prespecified features on the radiograph (nonsearch). Search situations leave the radiologist free 
to decide which and how many of the features on a particular radiograph he reports as abnormal. Nonsearch situations severely constrain the alternatives available to the radiologist, much as an observer's decisions are constrained in most laboratory perceptual tasks. The nonsearch tasks in the present experiments, which required an explicit judgment about each individual stimulus (test feature) in a set drawn from two distinct populations (targets and nontargets), used the same formal psychophysical procedure as a "yes-no" rating task. Nonsearch situations in clinical radiology frequently arise from specific questions posed during conferences or informal consultations about particular cases. These situations apparently place the radiologist at a disadvantage, at least in judging whether or not the radiographic feature in question represents actual pathology.

Although the model proposed in this paper is motivated by the problem of skilled visual search and applied to the detection of abnormal features on radiographs, the actual formulation is much more general. The formal detection model could be applied to analyze the performance of any "functionally serial" system that combines the decisions of two correlated detectors (one restricted to binary output), and makes a "positive" decision only if both separate detectors yield positive outputs. The particular simplifying assumptions and the methods used to estimate the model's parameters would depend upon the details of the specific application. Pollack and Madans (1964) considered a model defined by the concatenation of two independent detectors, whose decision variables were each represented by equalvariance normal distributions. This can be treated as a special case of the present model, in which $r_{t}=r_{n}$ $=0$ and $\sigma_{\mathrm{x}}=\sigma_{\mathrm{y}}=1.0 .^{5}$ Even for two independent detectors, Pollack and Madans show that the ROC curve of the serial detection system lies in between the separate ROC curves for the two detectors whenever there is sufficient mismatch in their individual detection capabilities.

\section{REFERENCE NOTE}

1. Swensson, R. G., Hessel, S. J., \& Herman, P. G. Radiographic interpretation with and without search: Visual search aids the recognition of chest pathology. Manuscript submitted for publication, 1979.

\section{REFERENCES}

Dorfman, D. D., \& AlF, E., JR. Maximum-likelihood estimation of parameters of signal-detection theory and determination of confidence intervals-rating-method data. Journal of Mathematical Psychology, 1969, 6, 487-496.
Green, D. M., \& Swets, J. A. Signal detection theory and psychophysics. New York: Wiley, 1966. (Reprinted: Huntington, N.Y: Krieger, 1974.)

GREY, D. R., \& Morgan, B. J. T. Some aspects of ROC curve-fitting: Normal and logistic models. Journal of Mathematical Psychology, 1972, 9, 128-139.

Harris, J. R., Shaw, M. L., \& Bates, M. Visual search of multicharacter arrays with and without gaps. Perception \& Psychophysics, 1979, 26, 69-84.

Herman, P. G., \& Hessel, S. J. Accuracy and its relationship to experience in the interpretation of chest radiographs. Invest $i$ gative Radiology, 1975, 10, 62-67.

Hoffman, J. E. Search through a sequentially presented visual display. Perception \& Psychophysics, 1978, 23, 1-11.

Kunde L, H. L., Nodine, C. F., \& CArmody, D. Visual scanning, pattern recognition and decision-making in pulmonary nodule detection. Investigative Radiology, 1978, 13, 175-181.

NeIsser, U. Cognitive psychology. New York: Appleton-CenturyCrofts, 1967.

Pollack, I., \& Madans, A. B. On the performance of a combination of detectors. Human Factors, 1964, 6, 523-531.

Sandor, T., \& Swensson, R. G. Evaluation of observer performance in detecting blood vessels on simulated angiographic images. Medical Physics, 1978, 5, 380-386.

Swensson, R. G., Hessel, S. J., \& Herman, P. G. Omissions in radiology: Faulty search or stringent reporting criteria? Radiology, 1977, 123, 563-567.

Swensson, R. G., Hessel, S. J., \& Herman, P. G. Detection performance and the nature of the radiologist's search task. In A. J. Alter (Ed.), Symposium on the optimization of chest radiography (Bureau of Radiological Health). Washington, D.C: U.S. Government Printing Office, 1979.

Zelen, M., \& Severo, N. C. Probability functions. In M. Abramowitz \& I. A. Stegun (Eds.), Handbook of mathematical functions (National Bureau of Standards). Washington, D.C: U.S. Government Printing Office, 1968.

\section{NOTES}

1. The variables $\mathrm{X}$ and $\mathrm{Y}$ are identifiable only to a linear transformation. This technical assumption simply picks a scale for $\mathrm{X}$ and $\mathrm{Y}$ that avoids introducing unnecessary parameters.

2. A Fortran IV program to generate the predicted ROC curve for any specified combination of the model's parameters is available from the author upon request. It approximates the infinite sum in Equation 1 by the first eight terms and generates a specified number of points over any designated range in $\mathbf{R}_{\mathbf{j}}$.

3. The similarity in the observed ROC curves for the free-search and focused-search conditions provides no basis for assuming that the value of $C$ changed between these two conditions. The observed differences can be attributed to shifts in $\mathbf{R}_{\mathrm{j}}$, which led to more reported target and nontarget test features at each level of confidence in the focused-search condition.

4. The fit could be improved by assuming that the correlation between the filter and evaluation processes is higher for target features than for nontarget features-e.g., $r_{t} \geqslant .7$ and $r_{n} \leqslant .2$.

5. In this special case, the predicted ROC curve for the joint detection system has only three parameters $\left(\Delta_{x}=d_{x}^{\prime}, \Delta_{y}=d_{y}^{\prime}\right.$, and $C)$ and is specified by a highly simplified form of Equation 1: $\mathrm{L}(\mathrm{h}, \mathrm{k}, \mathbf{0})=\mathrm{Q}(\mathrm{h}) \mathrm{Q}(\mathrm{k})$.

(Received for publication August 24, 1979; revision accepted November 13,1979 .) 